Histoire Épistémologie Langage

\section{Histoire Épistémologie Langage}

42-1 | 2020

La grammaire arabe étendue

\title{
Ouvrages de collaborateurs d'HEL
}

Publications by associates of HEL

\section{(2) OpenEdition}

\section{Journals}

Édition électronique

URL : https://journals.openedition.org/hel/634

DOI : $10.4000 /$ hel.634

ISSN : $1638-1580$

Éditeur

Société d'histoire et d'épistémologie des sciences du langage

\section{Édition imprimée}

Date de publication : 28 septembre 2020

Pagination : 192-195

ISSN : 0750-8069

\section{Référence électronique}

"Ouvrages de collaborateurs d'HEL », Histoire Épistémologie Langage [En ligne], 42-1 | 2020, mis en ligne le 28 octobre 2021, consulté le 29 octobre 2021. URL : http://journals.openedition.org/hel/634 ; DOI : https://doi.org/10.4000/hel.634

Ce document a été généré automatiquement le 29 octobre 2021.

\section{(c) (i) (9)}

HEL is licensed under a Creative Commons Attribution-NonCommercial-NoDerivatives 4.0 International License 


\title{
Ouvrages de collaborateurs d'HEL
}

\author{
Publications by associates of HEL
}

\section{NOTE DE L'ÉDITEUR}

Les « collaborateurs d'HEL » sont les membres du laboratoire HTL ainsi que les membres du bureau de la SHESL. Leurs ouvrages ne peuvent donner lieu à compte rendu dans HEL. / "Associates of HEL" refers to members of HTL research unit and to members of the SHESL board. Their publications shall not be reviewed in HEL.

1 Sablayrolles, Jean-François. 2020. Comprendre la néologie : conceptions, analyses, emplois. Limoges : Lambert-Lucas. La Lexicothèque. 312 p. ISBN 978-2-35935-286-3.

La néologie, phénomène universel des langues vivantes, touche pratiquement tous les domaines des sciences du langage et son traitement varie selon les modèles linguistiques empruntés (saussurien, martinetien, chomskyen...). Ce livre a pour objectif de proposer un tour d'horizon aussi complet que possible de ces questions et des réponses qui y sont apportées. Trois grandes parties exposent successivement: $\left(1^{\circ}\right)$ les concepts-clefs des créations lexicales avec leurs évolutions, leurs définitions et leurs rapports avec les dictionnaires; $\left(2^{\circ}\right)$ les différentes typologies qui en ont été proposées et, après distinction entre configuration et matrice, un tableau raisonné des matrices suivi de l'examen de cas délicats ; $\left(3^{\circ}\right)$ les utilisations et les utilisateurs de la néologie considérés dans ses aspects énonciatifs, de politique linguistique et de relations sociales.

2 Bisconti, Valentina, De Angelis, Rossana et Curea, Anamaria, dir. 2020. Héritages, réceptions, écoles en sciences du langage: avant et après Saussure. Paris: Presses de la Sorbonne Nouvelle. 366 p. ISBN 978-2-37906-030-4.

L'ouvrage offre un panorama des études actuelles dans le domaine de l'histoire et de l'épistémologie des sciences du langage. Conçu en hommage à Christian Puech, qui n'a cessé de faire dialoguer la linguistique et d'autres sciences humaines, il propose un état des lieux de la linguistique, de ses rapports avec des disciplines telles que la grammaire, 
la sémiologie et la philosophie, et identifie la place qu'elle occupe actuellement dans les sciences humaines.

Épistémologie et historiographie de la grammaire et de la linguistique, impact de l'œuvre de Saussure, courants structuralistes: trente-et-un articles, rédigés par des spécialistes internationaux de l'histoire et de l'épistémologie des sciences du langage, portent sur les traditions linguistiques européennes et extra-européennes, couvrant une période qui va de l'Antiquité à nos jours.

3 Léonard, Jean Léo et Avilés González, Karla Janiré, dir. 2019. Didactique des «langues en danger" : recherche-action en dialectologie sociale = Pedagogía co-participativa y "lenguas en peligro" : propuestas de dialectología social en acción. Paris: Michel Houdiard Éditeur. 242 p. ISBN 978-2-35692-177-2.

Ce livre est un recueil de propositions pour une didactique des langues vulnérables ou en danger. Il apporte une pierre de touche aux méthodes et théories de la « revitalisation » de ces langues. Il se présente comme un kaléidoscope de méthodes et d'idées pour développer des ateliers de développement de ressources pédagogiques en langues autochtones (TERPLO), à partir de multiples ateliers d'écriture réalisés principalement par et avec des locuteurs d'une trentaine de langues mésoaméricaines, mais également sous d'autres latitudes, comme l'Estonie. Des langues (nahuatl, mazatec, zapotec, etc.), ou des variétés dialectales d'un domaine linguistique (võro, mulgi et une variété orientale d'estonien, de Kodavere) qui, malgré la distance géographique et typologique, partagent les processus de résistance et de résilience sociolinguistique investis par leurs locuteurs. Cette dynamique de résistance, mais aussi de création et d'invention, d'élaboration et d'affinement de leur lexique et de leurs grammaires à travers la pratique pédagogiquement orientée de l'écriture, se reflète dans chacun des ateliers analysés ici. Ces travaux co-participatifs, menés aux côtés de linguistes et d'anthropologues qui partagent leurs connaissances techniques et méthodologiques avec les instituteurs, les élèves des écoles et les étudiants des universités interculturelles, démontrent que l'écriture n'est pas un instrument appartenant exclusivement aux langues "dominantes", mais plutôt un outil de communication, un processus par lequel l'oralité reste certes un élément inhérent à la vitalité linguistique et donc de la pérennité de ces langues. L'écrit agit plutôt comme une ressource que comme une barrière. L'oralité et l'écriture, le transfert des connaissances, l'autonomisation, la dialectologie sociale et perceptuelle, la confrontation des points de vue (de l'expert et de l'usager), l'optimisme et le pessimisme constituent quelques-uns des axes qui soutiennent ces TERPLO, ou ateliers d'écriture didactique des «langues de tradition orale» ou de toute langue menacée, contribuant à la construction épistémologique, méthodologique et écologique de cette praxis éducative et sociolinguistique qu'est la revitalisation des langues « en danger ».

Colombat, Bernard et Lahaussois, Aimée, dir. 2019. Histoire des parties du discours. Louvain : Peeters. Orbis/Supplementa 46. xxii + 563 p. ISBN 978-90-429-3952-3.

Comment définir le nom? Qu'est-ce qu'un verbe? Faut-il faire du pronom une catégorie distincte du nom ? Pourquoi l'article est-il une catégorie reconnue seulement dans certaines langues? À partir de quel moment a-t-on fait de l'adjectif une classe de mots à part? Peut-on trouver des interjections dans toutes les langues? Y a-t-il des classes de mots universelles? Pourquoi le nombre de parties du discours varie-t-il 
d'une langue à l'autre ? C'est à ces questions et à quelques autres que cet ouvrage veut répondre, en présentant une histoire des classes de mots (les "parties du discours ») dans la tradition occidentale et dans deux traditions «orientales» : arabe et sanskrite. Son originalité est d'inscrire cette histoire dans le long terme, en partant des classes identifiées dans la tradition grammaticale gréco-latine et en examinant ensuite le devenir de ces classes dans la grammaire française et d'autres traditions européennes. Le volume comporte quatorze chapitres: le premier est consacré à une étude des parties du discours dans leur ensemble, avec leurs "accidents", c'est-à-dire les catégories linguistiques qui les affectent, le deuxième au mot et les dix suivants à chacune des classes de mots (nom, article, adjectif, pronom, verbe, participe, adverbe, préposition, conjonction, interjection). Les deux derniers chapitres traitent des traditions grammaticales arabe et sanskrite. L'ouvrage se termine par une copieuse bibliographie et par trois index (auteurs, langues, concepts).

Tran Duc Thao. La dialettica materialista della coscienza. Éd. par Jacopo D’Alonzo. Rome : Castelvecchi. Le Navi. 2019. 96 p. ISBN 9788832828047

Cos'è la coscienza? E qual è il metodo per studiarla? Con questo saggio, pubblicato tra il 1974 e il 1975 e qui compendiato da note critiche e una ricca bibliografia, Tran Duc Thao, filosofo vicino a Sartre e a Merleau-Ponty e figura chiave della fenomenologia francese del secondo dopoguerra, propone un'inedita metodologia per lo studio della coscienza. Questa passa per un progetto semiologico, fortemente debitore della tradizione marxista, il quale non manca di alcuni elementi di grande originalità, come la nozione di "linguaggio della vita reale". Sullo sfondo di un bilancio biografico e teorico del suo percorso intellettuale, che non risparmia critiche all'individualismo e all'idealismo, Thao accoglie le sfide provenienti dalle scienze (linguistica, psicologia, biologia, paleoantropologia, genetica) e rivendica il ruolo svolto dal corpo, dall'interazione pratica con il mondo reale, dal linguaggio e dalle relazioni sociali nella formazione della coscienza.

6 Binaghi, Francesco et Sartori, Manuel, dir. 2019. Fuṣhā écrit contemporain : usages et nouveaux développements. Marseille: Diacritiques Éditions. $271 \mathrm{p}$. ISBN 979-10-97093-04-4.

Suite au colloque qui s'est tenu à Aix-en-Provence en juin 2015, intitulé « Matériaux pour l'établissement de grammaires descriptives du fuṣhā écrit contemporain. Entre norme(s) et pratiques au cours des 50 dernières années ", ce recueil propose d'ouvrir la réflexion sur la langue arabe écrite contemporaine et, plus particulièrement, sur ce qui est réputé dans le monde arabe comme étant du fuṣhā écrit contemporain (Contemporary Written Fușhāa - CWF).

À partir de données nouvelles, en termes de faits de langue, provenant d'un travail sur corpus écrits (littérature, presse, blogs, etc.) issus de divers supports (papier ou internet), ce recueil propose l'analyse de quelques aspects de cet arabe écrit contemporain. L'objectif est de rendre compte des évolutions notables de cette variété de langue, qui intéressent au premier chef la syntaxe, mais qui relèvent tout aussi d'évolutions sémantiques et d'usages sociolinguistiques.

7 Aussant, Émilie et Lahaussois, Aimée, dir. 2019. "Grammaires étendues » et descriptions de morphologie verbale (numéro thématique). Faits de langues 50/2. 
Leiden : Brill. 166 p. ISSN 1244-5460.

Cette livraison de Faits de langues rassemble quelques-uns des travaux présentés lors d'une journée d'étude consacrée aux «retombées » du phénomène des « Grammaires étendues » en linguistique descriptive, organisée en novembre 2016 avec le soutien du Labex EFL. Cet ensemble d'articles, rédigés par des linguistes descriptivistes qui s'interrogent sur les modèles grammaticaux utilisés, au cours de l'histoire, pour la description des langues ou des aires sur lesquelles ils travaillent, est éclairant à - au moins - deux titres : il connecte les pratiques actuelles avec l'histoire des descriptions, faisant émerger, pour une langue ou un sous-groupe de langues, l'évolution des termes et représentations utilisés; il montre, à ceux qui travaillent «au présent ", toute l'utilité des descriptions des langues réalisées par le passé. Outre les données que ces descriptions rassemblent, qui intéressent les descripteurs pour la dimension diachronique qu'elles donnent à voir, elles prouvent à quel point le «bricolage » des prédécesseurs est riche d'enseignements, à bien des égards. 